\title{
Hanani-Tutte and Monotone Drawings
}

\author{
Radoslav Fulek ${ }^{1, \star}$, Michael J. Pelsmajer ${ }^{2, \star \star}$, \\ Marcus Schaefer ${ }^{3}$, and Daniel Stefankovič ${ }^{4}$ \\ 1 Ecole Polytechnique Fédérale de Lausanne, Lausanne, Switzerland \\ radoslav.fulek@epfl.ch \\ 2 Illinois Institute of Technology, Chicago, IL 60616, USA \\ pelsmajer@iit.edu \\ 3 DePaul University, Chicago, IL 60604, USA \\ mschaefer@cs . depaul . edu \\ 4 University of Rochester, Rochester, NY 14627, USA \\ stefanko@cs.rochester.edu
}

\begin{abstract}
A drawing of a graph is $x$-monotone if every edge intersects every vertical line at most once and every vertical line contains at most one vertex. Pach and Tóth showed that if a graph has an $x$-monotone drawing in which every pair of edges crosses an even number of times, then the graph has an $x$-monotone embedding in which the $x$-coordinates of all vertices are unchanged. We give a new proof of this result and strengthen it by showing that the conclusion remains true even if adjacent edges are allowed to cross oddly. This answers a question posed by Pach and Tóth. Moreover, we show that an extension of this result for graphs with non-adjacent pairs of edges crossing oddly fails even if there exists only one such pair in a graph.
\end{abstract}

\section{Introduction}

The classic Hanani-Tutte theorem states that if a graph can be drawn in the plane so that no pair of independent edges crosses an odd number of times, then it is planar 6 619. (Two edges are independent if they do not have a shared endpoint.) There are many ways to look at this result; for example, in algebraic topology it is seen as a special case of the van Kampen-Flores theorem 9, Chapter 5] which classifies obstructions to embeddability in topological spaces. This point of view leads to challenging open questions (see, for example, [10]), but even in 2-dimensional surfaces the problem is not understood well (see [18 for a survey of what we do know).

Here, we study a variant of the problem for $x$-monotone drawings which was introduced by Pach and Tóth [12. A drawing of a graph is $x$-monotone if every edge intersects every vertical line at most once and every vertical line contains 
at most one vertex. The natural analogue of the Hanani-Tutte theorem in this context would state that every $x$-monotone drawing in which no pair of independent edges crosses an odd number of times has an $x$-monotone embedding, that is, a crossing-free drawing - without moving the vertices. The truth of this result was left as an open problem by Pach and Tóth.We prove it as Theorem 2 in Section 3. The extension of this result in the spirit of [1115] is not possible, which is proved in Section 4

The weak version of the classic Hanani-Tutte theorem states that if a graph can be drawn so that no pair of edges crosses oddly, then it is planar. The analogue for $x$-monotone drawings states that there is an $x$-monotone embedding if there is an $x$-monotone drawing in which no pair of edges crosses an odd number of times. This variant of the weak Hanani-Tutte theorem was first proved by Pach and Tóth 1 We give a new proof of this result as Theorem 11 in Section 2. which continues an elementary topological approach similar to earlier papers on the Hanani-Tutte theorem, e.g. [15.

A traditional approach to Hanani-Tutte style results is via obstructions; this sometimes leads to very slick proofs, like Kleitman's proof of the Hanani-Tutte theorem for the plane [7], but there are two drawbacks: complete obstruction sets are not always known, e.g. for the torus or, in spite of several attempts, for $x$-monotone embeddings (as discussed in [5]); and this approach is of little help algorithmically. Pach and Tóth took another approach, building on a proof of the weak Hanani-Tutte theorem for surfaces by Cairns and Nikolayevsky 2].

Before we begin, we introduce some basic terminology and notation. For any graph $G=(V, E)$ and $S \subseteq V(G)$, let $G[S]$ denote the subgraph induced by $S$; that is, the graph on vertex set $S$ with edge set $\{u v \in E(G): u \in S, v \in$ $S\}$. By a multigraph we understand a graph for which the set of edges is a multiset. A topological graph is a graph drawn in the plane where the vertices are represented by distinct points, and edges as Jordan arcs connecting the incident vertices, but not passing through any other vertex and any pair of edges crosses a finite number of times. Throughout the paper by a drawing of a graph we understand its representation as a topological graph. By an embedding of a graph we understand its (edge) crossing-free drawing.

The rotation at a vertex in a drawing of a graph is the clockwise ordering of edges at that vertex. The rotation system of a graph is the collection of rotations at its vertices. In an $x$-monotone drawing, the right (left) rotation is the clockwise order of the edges leaving the vertex towards the right (left). So (perhaps unfortunately), the right rotation is ordered from top to bottom, and the left rotation is ordered from bottom to top. We will not carefully distinguish between an abstract graph and a topological (drawn or embedded) graph, and "vertex" and "edge" are used in both contexts. We use $x(v)$ to denote the $x$ coordinate of a vertex $v$ located in the plane.

Remark 1. We are also preparing an extended version of this paper in which our results are used to solve algorithmic questions regarding level-planarity.

$\overline{1}$ There is a gap in the original argument; an updated version is now available $12[13$. 


\section{Weak Hanani-Tutte for Monotone Drawings}

An edge is even if it crosses every other edges an even number of times (including 0 times). A drawing is even if all its edges are even.

Theorem 1 (Pach, Tóth [12,13]). If $G$ has an $x$-monotone and even drawing, then $G$ has an $x$-monotone embedding in which each vertex keeps its $x$-coordinate and the rotation system remains unchanged.

Remark 2. The weak Hanani-Tutte theorem states that every graph with an even drawing is planar (without changing the rotation system). For background and variants of the weak Hanani-Tutte theorem, see [18.

Theorem 1 remains true if we require the resulting embedding to be straightline. This has nothing to do with the Hanani-Tutte part of the result; it is entirely due to the fact that any $x$-monotone embedding can be turned into a straight-line embedding in which every vertex keeps its $x$-coordinate 4 12. This redrawing can lead to an exponential blow-up in the area required for the drawing [8] (the examples in the paper allow multiple vertices in each layer, but these can be replaced by the requirement that vertices are not too close to edges they are not incident to).

Theorem 1 may prompt the reader familiar with Hanani-Tutte style results (in particular [11, Theorem 1] and [15, Theorem 2.1]) to ask whether something stronger is true: a "removing even crossings" lemma which would say that all even edges can be made crossing-free in the drawing of a graph which contains odd edges (while maintaining monotonicity). We will see in Section 4 that there cannot be any such lemma for monotone drawings.

Nearly the same result is claimed by Pach and Tóth in [12, Theorem 1.1], but instead of maintaining the rotation, Pach and Tóth state that we can find an equivalent $x$-monotone embedding for a given $x$-monotone and even drawing of $G$, where two drawings are equivalent if no edge changes whether it passes above or below a vertex. However, there are simple examples that show that one cannot hope to maintain equivalence in this sense, see Figure 1. The example can easily be turned into a 2-connected graph by replacing edges and vertices with cycles, so equivalence cannot be obtained by assuming 2-connectedness. On the other hand, see Corollary 1 for a positive result.

The original proof by Pach and Tóth contains a gap: it is not immediately clear how multiple faces that share a boundary can be embedded simultaneously 2 Filling in the details of this gap requires dropping equivalence. Pach and Tóth have prepared an updated version of the paper that includes a more detailed argument 13$]^{3}$

${ }^{2}$ In the text after Lemma 2.1 on page 42 of [12], $D_{\kappa}$ cannot necessarily be glued together without changing equivalence.

${ }^{3}$ In this newer version, equivalence is redefined to mean having the same rotation system. 


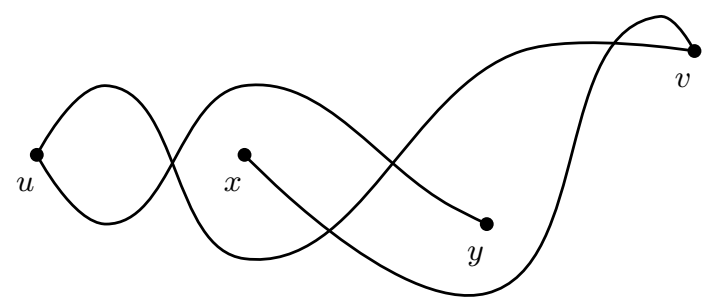

Fig. 1. $y$ lies above $x v$ and $x$ lies above $u v$. So in any equivalent $x$-monotone embedding with the same relative $x$-order of the vertices, $x v$ lies above $u v$, forcing $y$ above $u v$; but this contradicts $y$ being below $u v$.

We approach Theorem 1 in the spirit of earlier papers on the Hanani-Tutte theorem, e.g. [15. The proof, which is omitted, repeatedly makes use of a simple topological observation: suppose we are given two curves (not necessarily monotone) starting at $x=x_{1}$ and ending at $x=x_{2}$ which lie entirely between $x_{1}$ and $x_{2}$. The two curves cross an even number of times if and only if they have the same vertical order at $x=x_{1}$ and $x=x_{2}$ (if they start or end in the same point the vertical order at $x$ is determined by the order in which they enter $x$ ).

We will also find the following redrawing tool useful.

Lemma 1. Suppose a multigraph $G$ has an $x$-monotone embedding and let $f$ be an inner face of the embedding, with $m_{f}$ and $M_{f}$ being the leftmost and the rightmost vertex of $f$. If we add an edge $m_{f} M_{f}$ so that $m_{f} M_{f}$ lies in $f$, then the resulting graph $G \cup\left\{m_{f} M_{f}\right\}$ has an x-monotone embedding in which the relative $x$-order of the vertices and the rotation system remain the same. Note that we do not require $m_{f} M_{f}$ to be $x$-monotone initially and that there may be multiple ways of inserting $m_{f} M_{f}$ into the rotations at its endpoints.

Note that while the redrawing in Lemma 1 keeps the rotation system the same, it will destroy equivalence in the sense of Pach and Tóth. Indeed, this is necessarily the case as witnessed by a z-shaped corridor as the face in question:

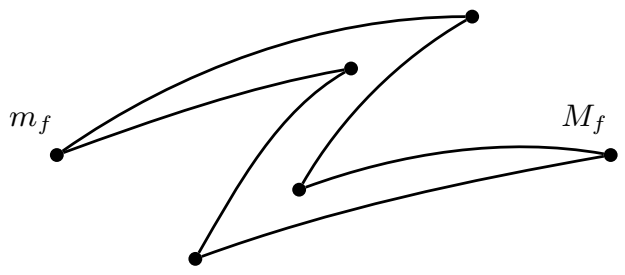

Fig. 2. Adding a monotone edge $m_{f} M_{f}$ into the corridor requires destroying equivalence 
Proof (of Lemma 11). If $G$ consists of multiple components, it is sufficient to prove the result for the component containing $f$ and shift its embedding vertically so that it does not intersect any other component. This allows us to assume that $G$ is connected. Then every face is bounded by a closed walk 4 The boundary of $f$ can be broken into two $m_{f}, M_{f}$-walks, $B_{1}, B_{2}$ with $B_{1}$ starting above $m_{f} M_{f}$ in the rotation at $m_{f}$, and $B_{2}$ starting below.

Let $D_{f}$ be the drawing of $G$ intersected with $U_{f}:=\left\{(x, y) \in \mathbb{R}^{2}: x\left(m_{f}\right)<\right.$ $\left.x<x\left(M_{f}\right)\right\}$. ( $D_{f}$ is a subset of the plane, not a graph.) We will locally redraw $G$ in $U_{f}$ so that $m_{f} M_{f}$ can be inserted as a straight-line segment. For each (topologically) connected component $Z$ of $D_{f}$, either $(i)$ for every $x$ between $x\left(m_{f}\right)$ and $x\left(M_{f}\right)$, there is a $y$-value of $B_{1}$ at $x$ that is below all $y$-values of $Z$ at $x$, or $(i i)$ for any $x$ between $x\left(m_{f}\right)$ and $x\left(M_{f}\right)$, there is a $y$-value of $B_{2}$ at $x$ that is above all $y$-values of $Z$ at $x$.

Let $Z_{1}$ be the union of all components of the first type, and $Z_{2}$ be the union of all components of the second type. Let $L$ be the line through $m_{f}$ and $M_{f}$. We will show how to move $Z_{1}$ to the half-plane above $L$, without changing the $x$-value of any point in $Z_{1}$ while fixing the points on the boundary of $U_{f}$. Let $P$ be an $x$-monotone curve with endpoints $m_{f}$ and $M_{f}$ that lies strictly below $Z_{1}$ in $U_{f}$ (note that $m_{f}$ and $M_{f}$ do not belong to $U_{f}$ ). Now move every point $v$ of $Z_{1}$ up by the vertical distance between $P$ and $L$ at $x=x(v)$. We proceed similarly to move $Z_{2}$ strictly below $L$, at which point $L$ is the desired embedding of $m_{f} M_{f}$.

In the proof of Theorem 1, all redrawing steps maintain equivalence except for applications of Lemma 1. This part of the proof, however, only arises in the case that $G-\left\{v_{1}, \ldots, v_{i}\right\}$, where $v_{j}$ denote $j$-th leftmost vertex of $G$, is not connected. Hence, if we can make an assumption on $G$ so that this case never occurs, we can conclude that the resulting embedding is equivalent to the original drawing in the sense of Pach and Tóth. We already saw that 2-connectedness is not sufficient, however, another notion is: a graph in which the vertices are ordered is a hierarchy if every vertex except the rightmost one has an edge leaving it towards the right $[3]$.

Corollary 1. If $G$ has an $x$-monotone and even drawing and $G$ is a hierarchy, then $G$ has an equivalent $x$-monotone embedding in which each vertex keeps its $x$-coordinate and the rotation system remains unchanged.

The assumption in Theorem 1 can be weakened, somewhat surprisingly, replacing $x$-monotonicity of edges by a weaker notion. Let us say that an edge $u v$ in a drawing is bounded if every interior point $p$ of $u v$ satisfies $x(u)<x(p)<x(v)$. That is, an edge is bounded if it lies strictly between its endpoints; it need not be $x$-monotone within those bounds.

Lemma 2. Suppose we are given a drawing of a graph $G$ with a bounded edge e. Then e can be redrawn, without changing the remainder of the drawing or the

${ }^{4}$ Walks are like paths except that vertices and edges can be repeated. In a closed walk the last vertex is the same as the first vertex. 
position of $e$ in the rotations of its endpoints, so that $e$ is $x$-monotone and the parity of crossing between $e$ and any other edge of $G$ has not changed.

Proof. Suppose that $e=a b$ and let $v \in V(G)$ be an arbitrary vertex between $a$ and $b: x(a)<x(v)<x(b)$. Now $e$ has to cross the line $x=x(v)$ an odd number of times since it connects $a$ to $b$. In particular, $v$ splits $x=x(v)$ into two: a part which is crossed an odd number of times by $e$, and the other part which is crossed evenly. In a small neighborhood of $x=x(v)$ redraw $G$ by pushing all crossings of $e$ with $x=x(v)$ from the even side across $v$ to the odd side. Note that the odd side of $x=x(v)$ remains odd and there are no crossing with $e$ left on the even side. Moreover, the parity of crossing between $e$ and any other edge does not change since $e$ is moved an even number of times across $v$. Repeat this for all $v$ between $a$ and $b$; now $e$ only passes above or below each such $v$, never both. We can now deform $e$ into an $x$-monotone edge connecting $a$ and $b$, without having the edge pass over any vertices. Therefore, this deformation does not affect the parity of crossing between $e$ and any other edge, so we have found the redrawing required by the lemma.

In hindsight we see that the redrawing of $e$ is quite effective: for each vertex $v$ between $a$ and $b$ we only need to know whether $e$ passes oddly above or below it, and we can build a polygonal arc from $a$ to $b$ that passes each vertex on the odd side.

\section{Strong Hanani-Tutte for Monotone Drawings}

Pach and Tóth [12] wrote "It is an interesting open problem to decide whether [Theorem 1] remains true under the weaker assumption that any two nonadjacent edges cross an an even number of times." The goal of this section is to establish this result, which was also conjectured in [18].

Theorem 2. If $G$ has an $x$-monotone drawing in which every pair of independent edges crosses evenly, then $G$ has an $x$-monotone embedding in which each vertex keeps its $x$-coordinate.

Remark 3. As in the case of Theorem 1] the statement of Theorem 2 remains true if we only require edges to be bounded rather than $x$-monotone: simply redraw edges one at a time using Lemma 2, before applying Theorem 2 ,

Let $G=(V, E)$ and $G^{\prime}=\left(V^{\prime}, E^{\prime}\right)$ denote two graphs. We say that $G<G^{\prime}$, if $|V|<\left|V^{\prime}\right|$ or if $|V|=\left|V^{\prime}\right|$ and $|E|<\left|E^{\prime}\right|$. In the sequel we consider minimal counterexamples with respect to this relation. In a proof of the standard Hanani-Tutte theorem, it is obvious that a minimal counterexample has to be 2-connected, since embedded subgraphs can be merged at a cut-vertex. Unfortunately, the merge requires a redrawing that does not maintain monotonicity, so here we must use structural properties that are more tailored to $x$-monotone redrawings.

Lemma 3. Suppose that $G$ is a minimal counterexample to Theorem 2. Then: 


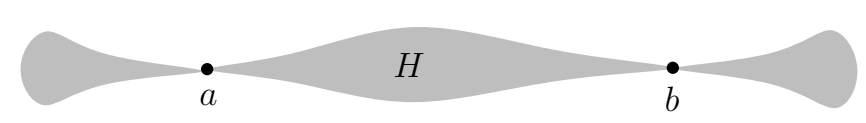

Fig. 3. Lemma 3(ii), forbidden subgraph $H$
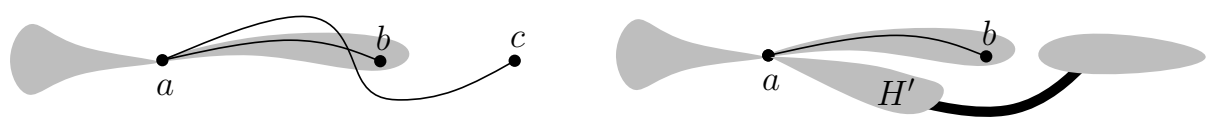

Fig. 4. Lemma 3(iii), forbidden edge $a c$ (left) and forbidden subgraph $H^{\prime}$ (right)

(i) $G$ is connected.

(ii) $G$ has no subgraph $H$ and vertices $a, b \in V(G) \backslash V(H)$ such that $x(a)<$ $x(v)<x(b)$ for all $v \in V(H), N(H)=\{a, b\}$, and $V(G) \backslash(V(H) \cup\{a, b\}) \neq$ $\emptyset$.

(iii) If $G$ has a cut-vertex a and $G-\{a\}$ has a component $H$ such that $x(a)<$ $x(v)$ for all $v \in V(H)$, then $H$ has only one vertex $b$, and $G$ has no edge ac with $x(b)<x(c)$. Also, in this case $G$ has no induced subgraph $H^{\prime} \neq \emptyset$ so that $x(a)<x(v)<x(b)$ for all $v \in V\left(H^{\prime}\right), a \in N\left(H^{\prime}\right) \neq\{a\}$, and $x(v)>x(b)$ for all $v \in N\left(H^{\prime}\right) \backslash\{a\}$.

Proof. If a minimal counterexample $G$ is not connected, none of its components are counterexamples to Theorem 2] But then we could embed each component separately and stack the drawings vertically so they do not intersect each other, yielding an embedding of $G$. This contradiction establishes $(i)$.

Consider case $(i i)$ (see Figure 3). Since $G$ is a minimal counterexample, both $G-V(H)$ and $G[V(H) \cup\{a, b\}]$ have embeddings (both graphs are smaller than $G$ by assumption). We can deform the crossing-free drawing of $G[V(H) \cup\{a, b\}]$ so that it becomes very flat. If $a b \in E(G)$ we can then insert this drawing into the drawing of $G-V(H)$ near the edge $a b$, without adding crossings. This gives us a crossing-free drawing of $G$, which is a contradiction. If $a b \notin E(G)$ then we add $a b$ to the drawing of $G-V(H)$ so that it has no independent odd crossings (we will presently see how this can be done); the resulting $G-V(H) \cup\{a b\}$ has fewer vertices than $G$ so it also has an embedding, and we can proceed as in the case that $a b \in E(G)$, removing the edge $a b$ in the end.

When $a b \notin E(G)$, here is how we draw the edge $a b$ with no independent odd crossings: Let $P$ be any $a, b$-path with interior vertices in $H$. By suppressing the interior vertices of $P$, we can consider it a bounded edge (in the sense defined earlier) between $a$ and $b$, so Lemma 2 tells us that we can draw an $x$-monotone edge that has the same parity of crossing with all edges of $G$ as does $P$.

Finally, we consider (iii) (see Figure 4), where $H$ is a component of $G-\{a\}$ so that $x(a)<x(v)$ for all $v \in V(H)$. If $|V(H)|>1$, let $b$ be the vertex with the largest $x$-value in $H$ and apply the part (ii) with $H:=H-b$. Since the previous proof requires that $H \neq \emptyset$, we are in the case that $V(H)=\{b\}$. If $G$ has an edge 
$a c$ with $x(b)<x(c)$, we can embed $G-\{b\}$ (since it is smaller than $G$ ), and then add $a b$ and $b$ to the embedding alongside of $a c$ without crossings.

It remains to consider an induced subgraph $H^{\prime} \neq \emptyset$ so that $x(a)<x(v)<x(b)$ for all $v \in V\left(H^{\prime}\right), a \in N\left(H^{\prime}\right) \neq\{a\}$, and $x(v)>x(b)$ for all $v \in N\left(H^{\prime}\right) \backslash$ $\{a\}$. By minimality, $G-\{b\}$ has an embedding. Consider the face which lies immediately below $H^{\prime}$; let $B$ be its boundary, and let $c$ be the vertex on $B$ with maximum $x$-value. $B$ is a closed walk that intersects $H^{\prime}$, and $H^{\prime}$ has edges to the left and right, so $B$ must contain neighbors of $H^{\prime}$ on its left and on its right. Therefore, $B$ contains $a$ (to the left of $H^{\prime}$ ), and by the choice of $H^{\prime}, x(c)>x(b)$. Using Lemma 1, we can add the edge $a c$ to the embedding of $G-\{b\}$ without introducing crossings. Since $x(a)<x(b)<x(c)$, we can instead add $a b$ to the drawing without crossings, so $G$ has an embedding which is a contradiction.

The proof of Theorem 2 now proceeds by induction on the number of odd pairs (pairs of edges that cross an odd number of times). Roughly speaking: If we encounter an odd pair (by necessity its edges are adjacent), we can either make it cross evenly or we are in a situation which has been excluded by Lemma 3. To realize this goal, we need more intermediate results. These results are not about minimal counterexamples, but are true in general.

For the lemmas we introduce some new terminology generalizing our usual notion of lying above or below a curve to curves with self-intersections: Let $C$ be a curve in the plane with endpoints $p$ and $r$ so that for every point $c \in C \backslash\{p, r\}$, $x(p)<x(c)<x(r)$. (This is similar to the definition of a bounded edge except that we allow self-intersections.) Suppose that $q$ is a point for which $x(p) \leq$ $x(q) \leq x(r)$. Extend $C$ via a horizontal ray from $p$ to $x=-\infty$ and a horizontal ray from $r$ to $x=\infty$, and consider the plane $\mathbb{R}^{2}$ minus that extended curve. We can 2-color its faces so that adjacent faces (faces whose boundaries intersect in a nontrivial curve) have opposite colors. We say that $q$ is above (below) $C$ if $q$ lies in a face with the same color as the upper (lower) unbounded region.

In the following two lemmas, let $G$ satisfy the assumption of Theorem 2 . that is, we assume that every pair of independent edges in $G$ crosses evenly. Both lemmas deal with the following scenario: $G$ contains three edges $e_{i}=v_{0} v_{i}$, $i \in\{1,2,3\}$ so that $e_{3}$ lies between $e_{1}$ and $e_{2}$ in the right rotation of $v_{0}$, with $e_{1}$ above $e_{2}$ at $v_{0}, e_{1}$ and $e_{2}$ cross oddly, and $e_{3}$ crosses each of the other two edges evenly.

Lemma 4. With an arbitrary vertex $v_{R}>x\left(v_{0}\right)$ define $G^{\prime}$ as the graph induced by $G$ on vertices $v$ with $x\left(v_{0}\right)<x(v) \leq x\left(v_{R}\right)$. Let $G_{i}^{\prime}$ be the component of $G^{\prime}$ that contains $v_{i}$. (If $x\left(v_{i}\right)>x\left(v_{R}\right)$, then $G_{i}^{\prime}=\emptyset$.)

If $G_{1}^{\prime}, G_{2}^{\prime}, G_{3}^{\prime}$ are pairwise disjoint and if for every $i$ there is a path $P_{i}$ from $v_{0}$ through $e_{i}$ to some vertex $v_{i}^{\prime}$ satisfying $x\left(v_{i}^{\prime}\right) \geq x\left(v_{R}\right)$ so that all vertices $v$ of $P_{i}$ satisfy $x(v) \geq x\left(v_{0}\right)$, then each $G_{i}^{\prime}$ has no neighbors (in $G$ ) to the left of $x\left(v_{0}\right)$, for $i \in\{1,2,3\}$.

Lemma 5. Suppose that for some distinct $j, k \in\{1,2,3\}$, there is a cycle $C$ that contains $e_{j}$ and $e_{k}$ such that every vertex $v$ of $C$ satisfies $x(v) \geq x\left(v_{0}\right)$. Let 
$v_{R}$ be the vertex on $C$ with largest $x$-value. Let $i$ be the unique index such that $\{i, j, k\}=\{1,2,3\}$. Suppose that $v_{i}$ is not in $C$.

Let $G_{i}^{\prime}$ be the component of $G-V(C)$ that contains $v_{i}$. Then every vertex $v$ of $G_{i}^{\prime}$ satisfies $x\left(v_{0}\right)<x(v)<x\left(v_{R}\right)$.

We are finally in a position to prove Theorem 2. We need one more piece of terminology: the distance between two edges $e, f$ is the number of edge ends between the ends of $e, f$ in the right (or left) rotation. (We do not measure distance within the entire rotation; only within the right or left rotation.)

Proof (of Theorem 2). Let $G$ be a minimal counterexample to the theorem. Fix a drawing of $G$ which minimizes the number of odd pairs, that is, the number of pairs of edges crossing oddly. If there are no odd pairs, then Theorem 1 completes the proof.

Suppose that there are edges $e_{1}$ and $e_{2}$ that cross oddly. Then $e_{1}$ and $e_{2}$ have a shared endpoint $v_{0}$, and we may assume that $v_{0}$ is the left endpoint of $e_{1}$ and $e_{2}$. Choose $e_{1}$ and $e_{2}$ so that their ends at $v_{0}$ have minimal distance in the right rotation at $v_{0}$, with $e_{1}$ above (that is, preceding) $e_{2}$. Then $e_{1}$ and $e_{2}$ are not consecutive in the rotation at $v_{0}$; if they were, they could be redrawn so that they cross once more near $v_{0}$, by switching their order in the rotation at $v_{0}$; this contradicts the choice of drawing of $G$. So there is at least one edge incident to $v_{0}$ that lies between $e_{1}$ and $e_{2}$ in the rotation at $v_{0}$, and by minimality, all such edges cross each other evenly and cross both $e_{1}$ and $e_{2}$ evenly. Pick one such edge, $e_{3}$. Let $v_{1}, v_{2}, v_{3}$ be the right endpoints of $e_{1}, e_{2}, e_{3}$, respectively, and let $G_{0}$ be the subgraph of $G$ induced by all vertices $v$ fulfilling $x(v) \geq x\left(v_{0}\right)$.

Case 1. The right endpoints of $e_{1}, e_{2}, e_{3}$ are in different components of $G_{0}-v_{0}$.

In Case 1 for each $i \in\{1,2,3\}$, consider the component of $G_{0}-v_{0}$ that contains $v_{i}$ and let $v_{i}^{\prime}$ be its vertex with largest $x$-value. Assign $i, j, k$ so that $\{i, j, k\}=$ $\{1,2,3\}$, and $x\left(v_{i}^{\prime}\right)$ is smaller than $x\left(v_{j}^{\prime}\right)$ and $x\left(v_{k}^{\prime}\right)$. Apply Lemma 4 with $x_{R}=$ $x\left(v_{i}^{\prime}\right)$, which defines $G_{i}^{\prime}, G_{j}^{\prime}, G_{k}^{\prime}$. By Lemma $3(i i i), G_{i}^{\prime}$ has only the one vertex $v_{i}=v_{i}^{\prime}$, and $G_{j}^{\prime}$ and $G_{k}^{\prime}$ are non-empty because $x\left(v_{i}\right)$ is greater than $x\left(v_{j}\right)$ and $x\left(v_{k}\right)$ (using $a=v_{0}, c \in\left\{v_{j}, v_{k}\right\}$ and $\left.b=v_{i}\right)$. Then we can apply the second part of Lemma 3 (iii) with $H^{\prime}$ equal to $G_{j}^{\prime}$ (or $G_{k}^{\prime}$ ) restricted to the vertices with $x$-coordinate smaller than $x\left(v_{i}^{\prime}\right)$, and we are done.

If we are not in Case 1] then let $v_{L}$ be the vertex with $x\left(v_{L}\right)$ chosen to be smallest such that the subgraph induced by vertices $v$ such that $x\left(v_{0}\right)<x(v) \leq$ $x\left(v_{L}\right)$ has a component that contains at least two right endpoints of $e_{1}, e_{2}, e_{3}$. Then there is a cycle $C$ that contains $e_{j}$ and $e_{k}$ for some distinct $k, j \in\{1,2,3\}$, and so that $x\left(v_{0}\right) \leq x(v) \leq x\left(v_{L}\right)$ for all $v \in V(C)$. If $v v_{L} \in\left\{e_{1}, e_{2}, e_{3}\right\}$, then we may assume that $C$ contains $v v_{L}$.

Let $i$ be the unique index for which $\{i, j, k\}=\{1,2,3\}$. By the previous assumption, $v_{i} \neq v_{L}$. By Lemma [5, $x\left(v_{i}\right)<x\left(v_{L}\right)$ or $v_{i} \in V(C)-v_{L}$.

Suppose that there is a path $Q$ from $v_{i}$ to $C$ so that $x\left(v_{0}\right)<x(v)<x\left(v_{L}\right)$ for all $v \in V(Q)$. Then $Q \cup e_{i} \cup C-v_{L}$ contains a cycle $C^{\prime}$ with $e_{i}$ and either $e_{j}$ or $e_{k}$. But every vertex $v$ of $C^{\prime}$ satisfies $x\left(v_{0}\right) \leq x(v)<x\left(v_{L}\right)$ for all $v$ in $C^{\prime}$, contradicting the choice of $v_{L}$. 
We can conclude that $v_{i}$ is not in $V(C)-v_{L}$, and if we let $G_{i}^{\prime}$ be the component of $G-V(C)$ that contains $v_{i}$, then $G_{i}^{\prime}$ has no neighbors in $V(C) \backslash\left\{v_{0}, v_{L}\right\}$. By Lemma 5 . $G_{i}^{\prime}$ lies between $x=x\left(v_{0}\right)$ and $x=x\left(v_{L}\right)$ (since $v_{i} \neq v_{L}$ ). Let $v_{i}^{\prime}$ be the vertex of $G_{i}^{\prime}$ with largest $x$-value. Apply Lemma 4 with $x_{R}=x\left(v_{i}^{\prime}\right)$. This defines $G_{i}^{\prime}, G_{j}^{\prime}, G_{k}^{\prime}$.

Case 2. $G_{i}^{\prime}$ is not adjacent to $v_{L}$.

(Same as Case 1) By Lemma 3 (iii), $G_{i}^{\prime}$ has only the one vertex $v_{i}=v_{i}^{\prime}$, and $G_{j}^{\prime}$ and $G_{k}^{\prime}$ are non-empty because $x\left(v_{i}\right)$ is greater than $x\left(v_{j}\right)$ and $x\left(v_{k}\right)$. Then we can apply Lemma $3(i i i)$ with $H^{\prime}$ equal to $G_{j}^{\prime}$ (or $G_{k}^{\prime}$ ) restricted to the vertices with the $x$-coordinate smaller than $x\left(v_{i}^{\prime}\right)$, and we are done.

Case 3. There is an edge from $G_{i}^{\prime}$ to $v_{L}$.

Apply Lemma 3( $(i i)$ with $H=G_{i}^{\prime}$. This completes the proof of the theorem.

\section{Monotone Crossing Numbers}

Our Hanani-Tutte results can be recast as results about monotone crossing numbers. For a leveled graph $(G, \ell)$ let mon-cr $(G, \ell)$ be the smallest number of crossings in any leveled drawing of $(G, \ell)$. Similarly, we can define mon-ocr $(G, \ell)$ as the smallest number of pairs of edges that cross oddly in any leveled drawing of $(G, \ell)$. Finally, mon-iocr $(G, \ell)$ is the smallest number of pairs of non-adjacent edges that cross oddly in any leveled drawing of $(G, \ell)$. We suppress $\ell$ and simply write mon-cr $(G)$, mon-ocr $(G)$, and mon-iocr $(G)$. With this notation we can restate the original result by Pach and Tóth, our Theorem 1 as saying that mon-ocr $(G)=0$ implies mon-cr $(G)=0$. Similarly, our Theorem 2 can be restated as mon-iocr $(G)=0$ implies mon-cr $(G)=0$.

From this point of view we can now ask questions that parallel analogous problems for the regular (non-monotone) crossing number variants: cr, ocr, and iocr. For example, we know that $\operatorname{ocr}(G)=\operatorname{cr}(G)$ for $\operatorname{ocr}(G) \leq 3[16$ and $\operatorname{iocr}(G)=$ $\operatorname{cr}(G)$ for $\operatorname{iocr}(G) \leq 2$ [17]. Pach and Tóth showed that $\operatorname{cr}(G) \leq\left(\begin{array}{c}\operatorname{ocr}(G) \\ 2\end{array}\right)[1115]$. The core step in this result is a "removing even crossings" lemma, in this particular case: if $G$ is drawn in the plane and $E_{0}$ is the set of its even edges, then $G$ can be redrawn so that all edges in $E_{0}$ are free of crossings. It immediately implies $\operatorname{cr}(G) \leq\left(\begin{array}{c}\operatorname{ocr}(G) \\ 2\end{array}\right)$, since only non-even edges can be involved in crossings (and every pair of non-even edges needs to cross at most once). A similar result for monotone drawings fails dramatically. In other words: even if there are only two edges crossing oddly and all other edges are even, then any $x$-monotone drawing of $G$ with the given leveling may require an arbitrary number of crossings. Thus we cannot hope to establish a "removing even crossings" lemma in the context of $x$-monotone drawings since it would imply a bound on mon-cr $(G)$ in terms of $\operatorname{mon}-\operatorname{ocr}(G)$.

Theorem 3. For every $n$ there is a graph $G$ so that $\operatorname{mon}-\operatorname{cr}(G) \geq n$ and $\operatorname{mon}-\operatorname{ocr}(G)=1$. 


\section{Open Questions}

We want to suggest some future avenues of research.

Monotone Crossing Numbers. The monotone crossing number of a leveled graph $G$ is the smallest number of crossings in any $x$-monotone drawing of the leveled graph. This problem is known to be NP-hard (even for two levels) and the monotone crossing number can be arbitrarily large, even for a planar graph (consider nested $>$ s). We get a more interesting question if we define the monotone crossing number for unleveled graphs as the smallest crossing number of any $x$-monotone drawing for any leveling of the graph. Is this monotone crossing number bounded in the crossing number? For comparison, $\operatorname{rcr}_{2}(G)$ is at most $\left(\begin{array}{c}\operatorname{cr}(G) \\ 2\end{array}\right)$, where $\operatorname{rcr}_{2}(G)$ allows straight-line edges with one bend [1]. Pach and Tóth in 14 recently proved that monotone crossing number for unleveled graphs is at most $\left(\begin{array}{c}\operatorname{cr}(G) \\ 2\end{array}\right)$. Then one can ask how far is this bound from the truth?

Bi-monotonicity. Let us define $y$-monotonicity like $x$-monotonicity after a 90 degree rotation; not very exciting by itself, but what happens if we want embeddings that are bi-monotone, that is, both $x$ - and $y$-monotone?

- If a graph has both an $x$-monotone embedding and a $y$-monotone embedding, does it always have a bi-monotone embedding?

- If there is a drawing of a graph which is bi-monotone, is there a straightline drawing with the same $x$ and $y$ ordering?

- What about bi-level-planarity?

As far as we know, bi-monotonicity and bi-level-planarity are new concepts, however, they are quite natural: If we specify the relative locations of objects on a map, we specify them in terms of "west/east of" and "north/south of" which is exactly what bi-monotonicity models. Imagine specifying the stations for a subway map: actual distance do not matter, what matters is relative location in terms of $x$ and $y$.

\section{References}

1. Bienstock, D., Dean, N.: Bounds for rectilinear crossing numbers. J. Graph Theory $17(3), 333-348(1993)$

2. Cairns, G., Nikolayevsky, Y.: Bounds for generalized thrackles. Discrete Comput. Geom. 23(2), 191-206 (2000)

3. Di Battista, G., Nardelli, E.: Hierarchies and planarity theory. IEEE Trans. Systems Man Cybernet. 18(6), 1035-1046 (1988, 1989)

4. Eades, P., Feng, Q., Lin, X., Nagamochi, H.: Straight-line drawing algorithms for hierarchical graphs and clustered graphs. Algorithmica 44(1), 1-32 (2006)

5. Estrella-Balderrama, A., Fowler, J.J., Kobourov, S.G.: On the Characterization of Level Planar Trees by Minimal Patterns. In: Eppstein, D., Gansner, E.R. (eds.) GD 2009. LNCS, vol. 5849, pp. 69-80. Springer, Heidelberg (2010)

6. Chojnacki, C., (Hanani, H.).: Über wesentlich unplättbare Kurven im dreidimensionalen Raume. Fundamenta Mathematicae 23, 135-142 (1934) 
7. Kleitman, D.J.: A note on the parity of the number of crossings of a graph. J. Combinatorial Theory Ser. B 21(1), 88-89 (1976)

8. Lin, X., Eades, P.: Towards area requirements for drawing hierarchically planar graphs. Theor. Comput. Sci. 292(3), 679-695 (2003)

9. Matoušek, J.: Using the Borsuk-Ulam theorem. Universitext. Springer, Berlin (2003); Lectures on topological methods in combinatorics and geometry, Written in cooperation with Anders Björner and Günter M. Ziegler

10. Matousek, J., Tancer, M., Wagner, U.: Hardness of embedding simplicial complexes in $\mathbb{R}^{d}$. In: Mathieu, C. (ed.) Proceedings of the Twentieth Annual ACM-SIAM Symposium on Discrete Algorithms, SODA 2009, New York, NY, USA, January 4-6, pp. 855-864. SIAM (2009)

11. Pach, J., Tóth, G.: Which crossing number is it anyway? J. Combin. Theory Ser. B 80(2), 225-246 (2000)

12. Pach, J., Tóth, G.: Monotone drawings of planar graphs. J. Graph Theory 46(1), 39-47 (2004)

13. Pach, J., Tóth Monotone, G.: Drawings of planar graphs. ArXiv e-prints (January 2011)

14. Pach, J., Tóth, G.: Monotone crossing number. In: Graph Drawing (to appear, 2011)

15. Pelsmajer, M.J., Schaefer, M., Štefankovič, D.: Removing even crossings. J. Combin. Theory Ser. B 97(4), 489-500 (2007)

16. Pelsmajer, M.J., Schaefer, M., Štefankovič, D.: Odd crossing number and crossing number are not the same. Discrete Comput. Geom. 39(1), 442-454 (2008)

17. Pelsmajer, M.J., Schaefer, M., Štefankovič, D.: Removing independently even crossings. SIAM Journal on Discrete Mathematics 24(2), 379-393 (2010)

18. Schaefer, M.: Hanani-Tutte and related results. To appear in Bolyai Memorial Volume

19. Tutte, W.T.: Toward a theory of crossing numbers. J. Combinatorial Theory 8, 45-53 (1970) 